\title{
THE DEFORMATION AND SHEAR BANDS IN THE Fe-3\%Si ALLOY
}

\author{
B.K. SOKOLOV *, V.V. GUBERNATOROV, \\ I.V. GERVASYEVA, A.K. SBITNEV and L.R. VLADIMIROV \\ Institute of Metal Physics, Ural Division of Russian Academy of Sciences, \\ 18 Kovalevskaya street, 620219, Ekaterinburg, Russia
}

(Received in final form 28 September 1997)

\begin{abstract}
This paper deals with a metallographic study of shear and deformation bands, which are formed and developed during rolling and fatigue tests of single crystals and polycrystals of $\mathrm{Fe}-3 \% \mathrm{Si}$. The effect of the initial orientation of the single crystals, dimensions of grains in polycrystals, thickness of the samples and certain rolling factors on the formation of banded structures was analyzed. The effect of these structures on the structure and the texture, which appear in the deformed alloy subject to annealing, was studied too.

The obtained data provided a better account of the banded structures and permitted directing ways to control their formation and evolution.
\end{abstract}

Keywords: Rolling; Deformation bands; Shear bands; Texture

\section{INTRODUCTION}

It has long been known that plastic deformation of materials is nonuniform. A manifestation of this nonuniformity is the appearance of banded features: deformation bands and shear bands. These bands may pass across grain boundaries and reach mesoscopic (within groups of grains) or macroscopic (within the whole sample) dimensions. They tell upon the processes of subsequent plastic deformation and failure of materials, the formation of the deformation texture, the structural state and the annealing texture of materials, thus determining their final properties.

\footnotetext{
* Corresponding author.
} 
The following is known about the shear bands:

- They are formed in various materials - metals and rocks, ionic crystals and organic compounds - subject to all types of plastic deformation. The degree of plastic deformation, $\varepsilon$, which gives rise to the bands, depends on the particular material and ranges from 0.01 to 3 or higher.

- The bands were not detected in deformed single crystals of copper

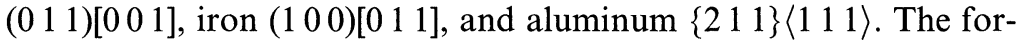
mation of the bands is suppressed in well-annealed pure metals. Chemical and structural inhomogeneities, impurities and inclusions facilitate the formation of the bands.

- The bands appear as equal-width regions having clearly defined boundaries. As the band develops, the matrix orientation changes abruptly or gradually towards the middle of the band.

- The bands have a certain direction relative to the acting force. Their traces are perpendicular to the direction of this force on the deformed surface of the sample (for example, rolling surface) and go at an angle of $15-55^{\circ}$ (in most cases at $35^{\circ}$ ) on the side surfaces of the sample. The bands cross the slip planes.

- The appearance of the bands is followed by the formation of a relief on the sample's surface.

Some fundamental issues of the nature of the bands have been so far unclear. Why do these bands appear? Why does the inclination angle of the bands to the acting force remain constant? What effects do the grain dimensions, thickness of the material and deformation factors have on the formation of the bands and their structure? What is the mechanism of the band formation (crystallographic, noncrystallographic, shear, kink)?

The issues relating to the appearance and development of the deformation bands have been no less complicated. The following is known:

- The deformation bands do not appear in crystals having stable orientations, which do not change under deformation.

- The boundaries between the deformation bands - transition bands comprise microbands whose orientation changes gradually from the orientation of one deformation band to that of another. These microbands include those having the initial orientation of the crystal. 
The debated issues are the origin of the deformation bands, the dependence of the bands on the change of the form of the material and alteration of the orientation, and the effect of the grain dimensions, thickness of the material and the deformation factors on the structure of the bands.

It is necessary to know how recrystallization nuclei are formed on the aforementioned-banded features and whether particular annealing conditions affect the location of nucleation sites in the bands.

The solution of these problems is very important in terms of devising new methods to control the formation of the structure and the texture of materials, model and predict the texture, the structure and properties of materials under various deformation and thermal conditions.

The literature concerned with the banded features has placed particular emphasis on materials having a limited number of slip systems (for example, HCP metals, ionic crystals, rocks), where the kink phenomena are thought to be the main reason for the formation of the bands. Numerous papers have been dedicated to FCC metals, which were used for a comprehensive study and modeling of the processes of the shear-induced formation of the bands under deformation.

We have examined the formation of the bands during plastic deformation of an iron-silicon alloy (BCC lattice), which has a large number of possible slip systems. The available treaties offer evidence that the formation of banded structures in such materials has certain specific features.

\section{MATERIALS AND METHODS}

Plate $(0.5 \mathrm{~mm}$ thick $)$ single crystals having different orientations were produced from a commercial $\mathrm{Fe}-3 \% \mathrm{Si}$ alloy using the secondary recrystallization and recrystallization after critical strain hardening. Polycrystals having grains of different dimensions were prepared by plastic deformation and annealing. The samples were cold-rolled on a laboratory mill to different degrees of deformation $(\varepsilon=0.05-3$.2). Some of the single crystals and polycrystals were subject to the multicycle cantilever simple bending fatigue test (the frequency of the symmetrical reversed cycles was $16-18 \mathrm{~Hz}$ ). 
The relief formed on the polished surface of the samples and the microstructure after etching in nithol and the Shostock reagent, which reveals dislocations in decorated samples, were examined.

The crystal orientation and the texture were studied by the pole figure method, using X-ray diffractometer with $\mathrm{CoK}_{\alpha}$ radiation. The etch pattern method was used to study the orientation and texture nonuniformities. This method, when the whole surface of the sample was covered with tiny $(2-3 \mu \mathrm{m})$ etch patterns, proved to be efficient. The orientation (texture) nonuniformity and its distribution could be determined from the variation of the intensity of the reflected light from different areas of the microsection.

\section{EXPERIMENTAL RESULTS}

\section{The Structure of the Deformed Single Crystals (1 110$)$}

Figure 1 shows the microstructures, which were detected in the orthogonal planes of the single crystal $(110)$ rolled with a $70 \%$ reduction in the direction [0 01 1]. Deformation bands aligned with the rolling direction and shear (kink) bands going across the rolling direction are seen in the rolling plane (RD-TD plane). The orientation of the adjacent deformation bands changes from $\left(\begin{array}{llll}1 & 1 & 1\end{array}\right)\left[\begin{array}{lll}1 & 1 & \overline{2}\end{array}\right]$ to $\left(\begin{array}{llll}1 & 1 & \overline{1}\end{array}\right)\left[\begin{array}{lll}1 & 1 & 2\end{array}\right]$. The metastable orientation $\left\{\begin{array}{llll}1 & 1 & 1\end{array}\right\}\left\langle\begin{array}{lll}1 & 1 & 2\end{array}\right\rangle$ is achieved by breaking the single crystal up into fragments and rotation of the lattices in these fragments clockwise and counter-clockwise about the transverse direction. It may be seen that on the transverse plane (TD-ND plane) the deformation bands do not pass all the way through the sample. The directions of the shear bands in side cross-sections (RD-ND plane) of the sample differ for the deformation bands (they are close to $+32^{\circ}$ in certain deformation bands and to $-32^{\circ}$ in adjacent deformation bands relative to the rolling direction).

The study of the formation of the banded structures revealed that the slip is nonuniform in single crystals starting from the very small degrees of deformation. The slip traces are usually detected by the relief formed on the polished surface of the sample. In the case of rolling with the reduction of $5-8 \%$ the slip traces may be revealed by etching. However, this treatment makes noticeable only the coarsest lattices having considerable distortions due to local rotations. In literature these coarse 


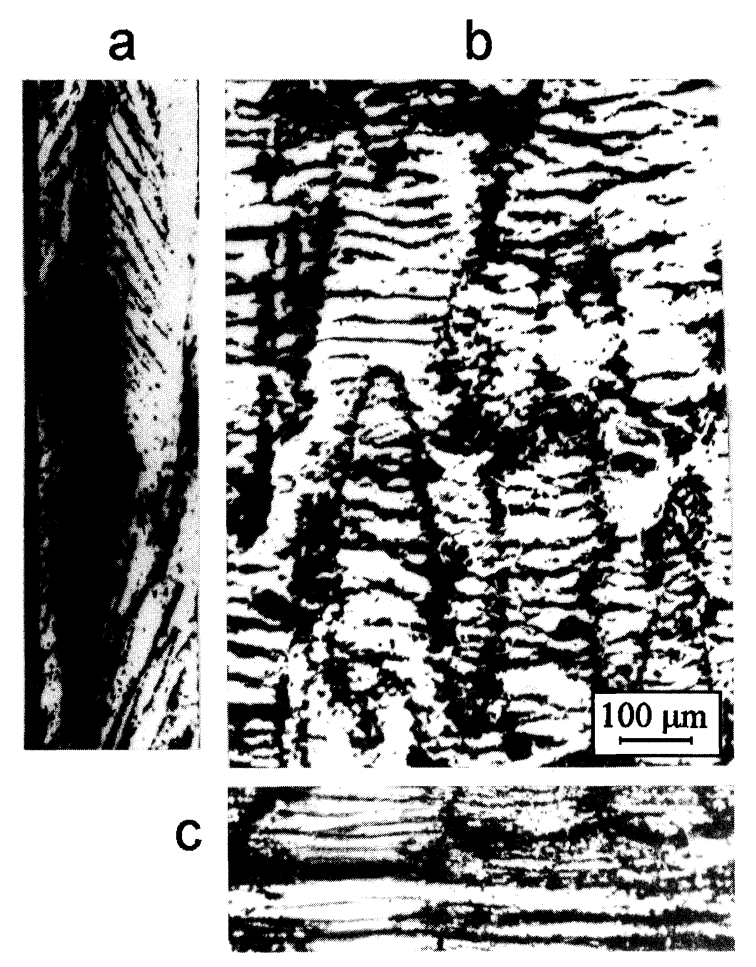

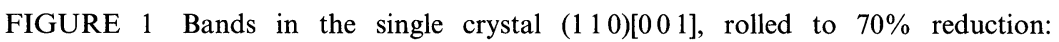
(a) RD-ND surface; (b) RD-TD surface; (c) TD-ND surface.

bands are termed the macroslip. Figure 2(a) illustrates the distribution of the said bands. They correspond to the conventionally described slip planes of the $\left\{\begin{array}{ll}1 & 10\end{array}\right\}$ type. Even at small degrees of deformation the initial orientation of the crystal varies considerably (up to $8^{\circ}$ ). At the deformation of $15-18 \%$ a clear-cut doublet of the orientations of the $\{331\}\langle 116\rangle$ type appears.

If the deformation is increased to $20 \%$, a structure comprising brightetched regions separated by dark-etched regions may be revealed by etching (Fig. 2(b)). The orientation of the bright regions corresponds to the general direction of the texture evolution during deformation. The dark regions contain bands, which are parallel to the transverse direction. Their plane is close to $\left\{\begin{array}{lll}1 & 1 & 2\end{array}\right\}$. The orientation of the lattice between the bands approximates the initial orientation. Even at these degrees of deformation shear bands are observed within the regions having an 

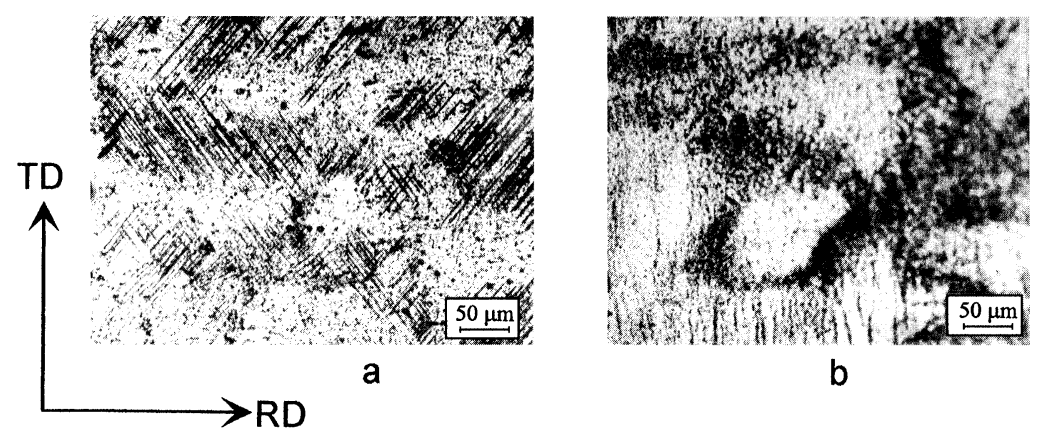

b

FIGURE 2 The begining of band structure formation in single crystals $\left(\begin{array}{lll}1 & 1 & 0\end{array}\right)\left[\begin{array}{lll}0 & 0 & 1\end{array}\right]$ : (a) the distribution of macroslip bands at rolling reduction $8 \%$; (b) appearance of initial deformation bands at rolling reduction $20 \%$.

altered orientation. Subsequent to rolling with a reduction of $20 \%$, the structural regions having the initial orientation narrow, are ordered, and turn into the transition bands. It is remarkable that the number of the deformation bands does not grow, while the number of the shear bands is enhanced considerably with the further increase in the degree of deformation. One more specific feature of the shear bands is worth noting: their number grows with decreasing width of the deformation bands.

If the single crystal $\left(\begin{array}{lll}1 & 1 & 0\end{array}\right)$ is rolled in the direction [ $\left[\begin{array}{ll}1 & 1\end{array}\right]$, the structural pattern is similar to its counterpart discussed above. Despite the fact that a one-component texture of the $\left\{\begin{array}{lll}2 & 1 & 1\end{array}\right\}\left\langle\begin{array}{lll}0 & 1 & 1\end{array}\right\rangle$ type is formed under large deformations, the deformed single crystal is broken up into fragments. In adjacent fragments the lattices rotate clockwise and counter-clockwise about the transverse direction. When the degree of reduction is increased, deformation bands separated by transition bands appear too. Shear bands develop within the deformation bands. During the texture evolution the plane of the shear bands changes from $\left\{\begin{array}{lll}1 & 1 & 0\end{array}\right\}$ to $\left\{\begin{array}{lll}2 & 1 & 1\end{array}\right\}$. In doing so, it passes $\left\{\begin{array}{lll}3 & 1 & 2\end{array}\right\}$.

When the single crystal $\left(\begin{array}{lll}1 & 1 & 0\end{array}\right)$ is rolled in the direction [ 110$]$, very wide, curved and branched shear bands are formed (Fig. 3). The angle between separate fragments of the bands and the transverse direction may vary from $+30^{\circ}$ to $-30^{\circ}$. In the side cross-sections the wide bands make an angle of $15-38^{\circ}$ with the rolling direction. In the transverse cross-section (ND-TD plane) their traces are nearly parallel to the transverse direction. It is difficult to assign any crystallographic indices 

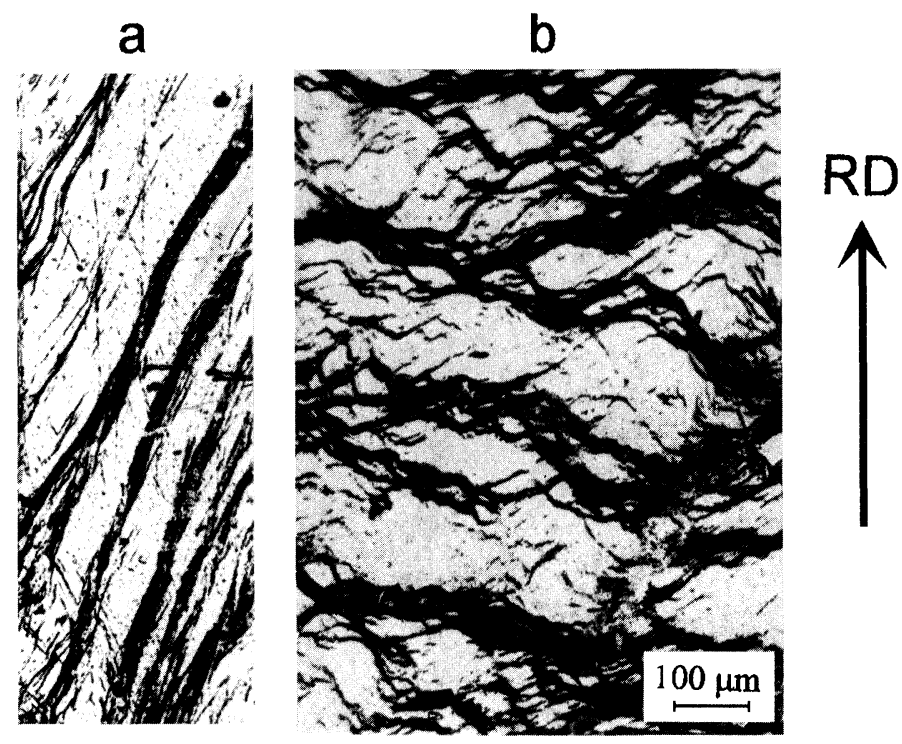

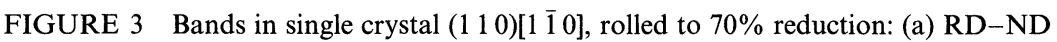
surface; (b) RD-TD surface.

to these shear surfaces. The bands may be assumed to be similar to $\left\{\begin{array}{lll}1 & 0 & 0\end{array}\right\}-\left\{\begin{array}{lll}3 & 1 & 0\end{array}\right\}$. Beginning of shear band formation is shown in Fig. 4.

The width of the single crystal increases to a large extent under deformation. The produced texture contains various components. The strongest component is close to (2 111$)\left[\begin{array}{lll}0 & \overline{1} & 1\end{array}\right]$. However, even if the texture has many components, deformation bands cannot be detected in the deformed crystal. But if the surface is treated to a fine etch pattern, it is seen that the whole single crystal is broken up into numerous different-orientation bands both in the transverse and longitudinal directions.

\section{The Structure of Deformed Single Crystals having Stable Orientations}

When the single crystal having the initial orientation (1 111$)$ is rolled in the direction [ $11 \overline{1} 0]$, its orientation should not change. This orientation is stable with respect to deformation. However, the pole figure exhibits a strong scattering of the weak component in the rolling direction 


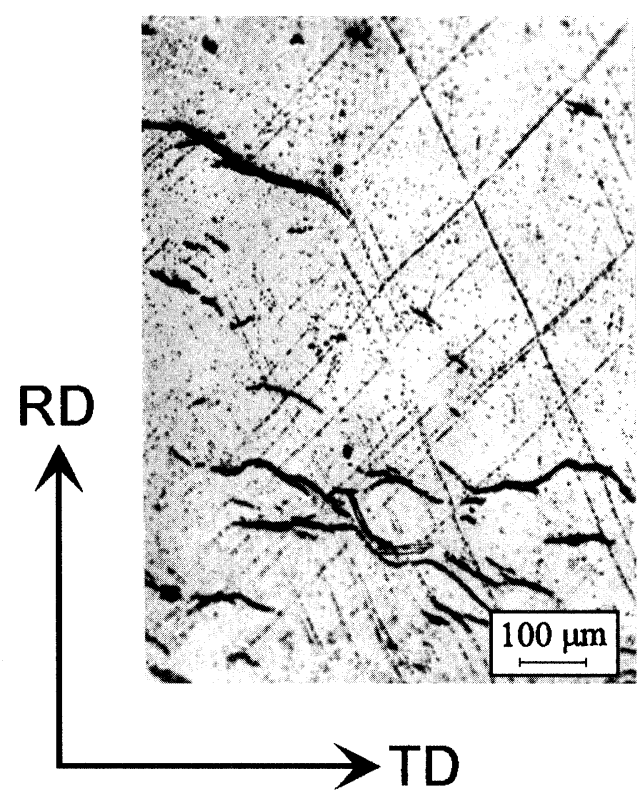

FIGURE 4 The appearance of initial shear bands in the single crystal (llll 110$)\left[\begin{array}{lll}1 & 1 & 0\end{array}\right]$, rolled to $17 \%$ reduction.

(Fig. 5(a)). Transverse bands are found in the deformed crystal (Fig. 5(b)). Treatment of the rolling plane to a fine etch pattern reveals a characteristic pattern (Fig. 5(c)), which consists of alternating bright and dark bands. This means that shear bands also develop in single crystals having stable orientations. It is these shear bands that are responsible for the scattering of the deformation texture.

The orientation of the crystal (100), which is deformed in the direction $\left[\begin{array}{lll}0 & 1 & 1\end{array}\right]$ remains almost unchanged, although weak components are scattered mainly in the transverse direction (Fig. 6(a)). Banding parallel to the rolling direction may be seen on the rolling surface bearing the etch pattern. Sometimes faint traces of banding appear in the transverse direction (Fig. 6(b)).

\section{The Structure of the Rolled Polycrystals}

Polycrystals having different initial mean dimensions of the grains were studied. The fine-grain $(19,23$ and $47 \mu \mathrm{m})$ samples had a multicomponent characteristic recrystallization texture dominated 

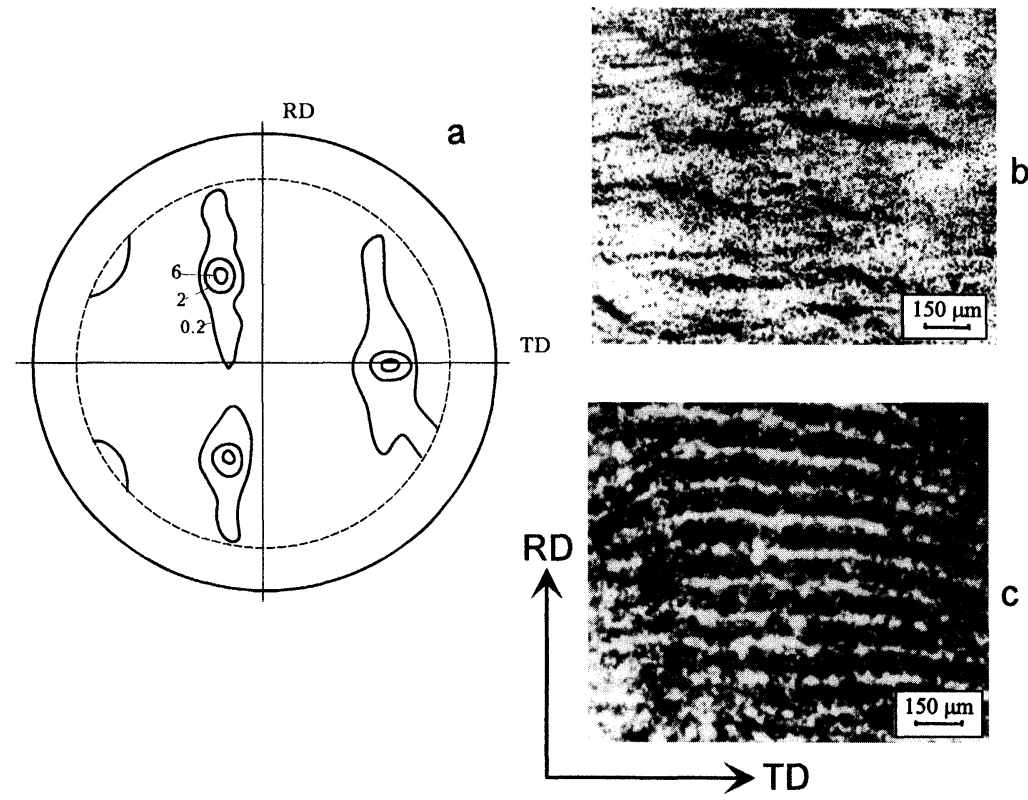

FIGURE 5 The texture and structure of the single crystal (1 111$)\left[\begin{array}{ll}1 & 1\end{array}\right]$, rolled to $75 \%$ reduction: (a) pole figure $\{110\}$; (b) bands in RD-TD surface; (c) RD-TD surface of deformed single crystals with etch-pit.
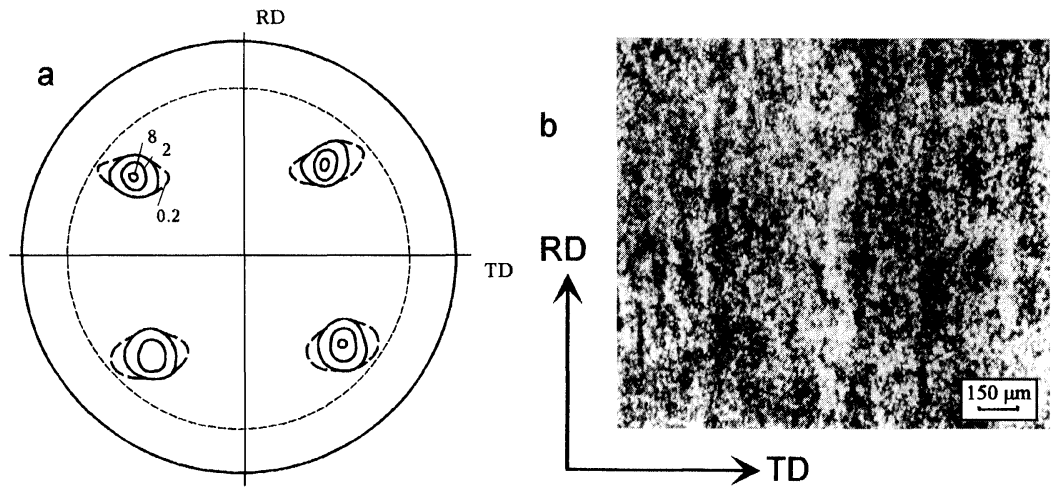

FIGURE 6 The texture and structure of the single crystal $(100)[011]$, rolled to $85 \%$ reduction: (a) pole figure $\{110\}$; (b) RD-TD surface of deformed single crystals with etch-pit. 


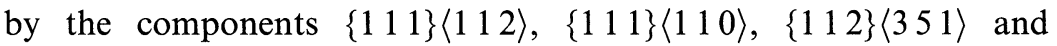
$\left\{\begin{array}{lll}1 & 0 & 0\end{array}\right\}\langle 031\rangle$. The texture of the coarse-grain $(140,660 \mu \mathrm{m}$ and $5 \mathrm{~mm})$ samples was largely scattered. The sample having a medium dimension (about $12 \mu \mathrm{m})$ of the grains and a very sharp texture (1 110$)\left[\begin{array}{lll}0 & 0 & 1\end{array}\right]$ were studied too.

Figure 7(a) shows the structure of the coarse-grain material after cold deformation to $70 \%$. The initial orientations of the grains were
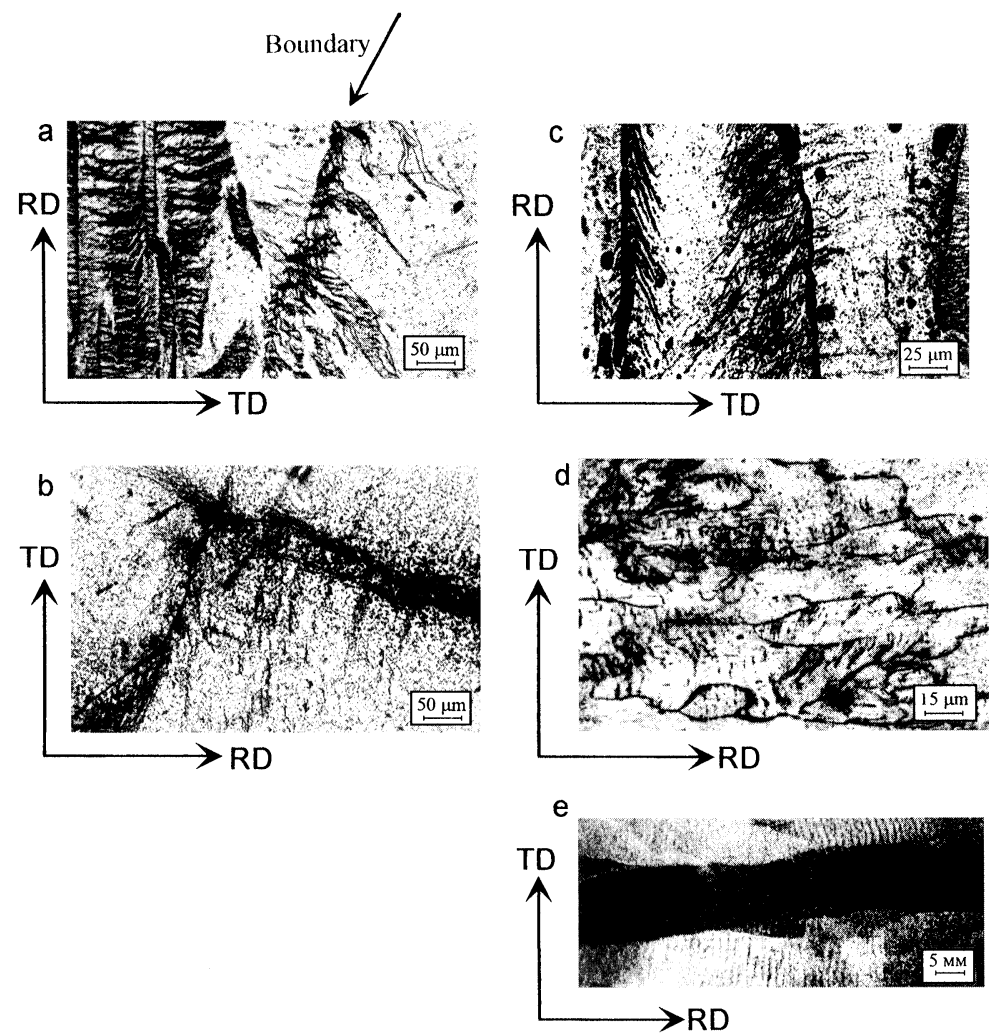

FIGURE 7 Bands in cold-rolled polycrystals with different initial grains size: (a)

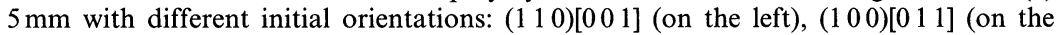
right). Bands break near the grain boundary. Rolling reduction 70\%; (b) the appearance of initial shear bands in coarse-grained $(5 \mathrm{~mm})$. Rolling reduction $20 \%$; (c) $140 \mu \mathrm{m}$. Rolling reduction $70 \%$; (d) $23 \mu \mathrm{m}$. Rolling reduction $70 \%$; (e) bands in highly textured polycrystals. Fine grains $(12 \mu \mathrm{m})$ are inside of initial coarse grains. Rolling reduction $70 \%$. 


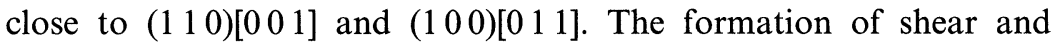
deformation bands within every grain during rolling obeys the same rules as those discussed above for the single crystals. However, the bands are strongly distorted in the boundary regions. In the grain having the initial orientation $\left(\begin{array}{llll}1 & 1 & 0\end{array}\right)\left[\begin{array}{lll}0 & 0 & 1\end{array}\right]$ the direction of the deformation bands changed and their number considerably decreased. The deformation bands appeared simultaneously in the boundary region of the grain $\left(\begin{array}{lll}1 & 0 & 0\end{array}\right)\left[\begin{array}{lll}0 & 1 & 1\end{array}\right]$. The distribution of the shear bands also changed to a large extent.

The different character of the banded structures observed in the boundary regions and the central areas of the crystals is due to the deformation crystallography. Even at a small deformation the slip in the boundary regions differs largely from the slip in the crystallites. When the degree of deformation is increased to $20 \%$, separate areas of shear bands extending from the boundaries appear (Fig.7(b)). One may see that in the deformed single crystals, too, the shear bands near the transition bands differ in their character from those within the deformation bands. A fringe of small shear bands may be seen near the transition band in the single crystal ( $\left(\begin{array}{lll}1 & 1 & 0\end{array}\right)\left[\begin{array}{lll}0 & 0 & 1\end{array}\right]$ deformed to $70 \%$. In the rolled polycrystals where the grains had the mean dimension of $140 \mu \mathrm{m}$ the etch pattern method revealed a highly nonuniform distribution of the orientations even within a grain. The deformation and shear bands exhibit an extremely nonuniform distribution (Fig. 7(c)). When the mean dimension of the initial grains equals $47 \mu \mathrm{m}$, the deformation nonuniformity is enhanced. The deformation bands decrease in number and become irregular. The rotation of the grains relative to one another gains in significance with decreasing mean dimension of the grains. In the samples having the mean grain dimensions of $19-23 \mu \mathrm{m}$ the rotations bear a regular character. Stable components of the $\left\{\begin{array}{llll}1 & 1 & 1\end{array}\right\}\langle 110\rangle$ and $\left\{\begin{array}{lll}1 & 0 & 0\end{array}\right\}\left\langle\begin{array}{lll}0 & 1 & 1\end{array}\right\rangle$ types are enhanced, while unstable and metastable components (for example, $\left\{\begin{array}{llll}1 & 1 & 1\end{array}\right\}\left\langle\begin{array}{lll}1 & 1 & 2\end{array}\right)$ ) diminish under deformation. The deformation due to the grain rotation and the grain-boundary slip decreases the tendency to the band formation. However, shear bands passing through groups of fine grains may be observed in certain cases (Fig. 7(d)). The plastic deformation of a fine-grain textured material is accompanied by the appearance of numerous shear bands (Fig. 7(e)). 


\section{The Change of the Structure of the Polycrystals During Fatigue Tests}

Shear bands are formed during fatigue tests too. A specific feature of these tests is that the material is deformed and even fails as a result of a large number of repeated loading cycles at a stress lower than the usual yield stress. The structure observed in the samples after $10^{6}$ loading cycles at a stress accounting for about $70 \%$ of the yield stress of the material in the initial state is shown below.

In the single crystals and the coarse-grain polycrystals shear bands were observed in the zone of the crack propagation (Fig. 8(a)).

The plastic deformation had a clearly pronounced local character in the fine-grain samples subject to the fatigue tests. The so-called extrusion areas appeared on the surface of the polished sample. A marked relief was formed in these areas. Longitudinal shear bands passing through groups of fine grains were seen in these areas (Fig. 8(b)), although deformation was due also to the grain-boundary slip and rotation of the grains relative to one another.

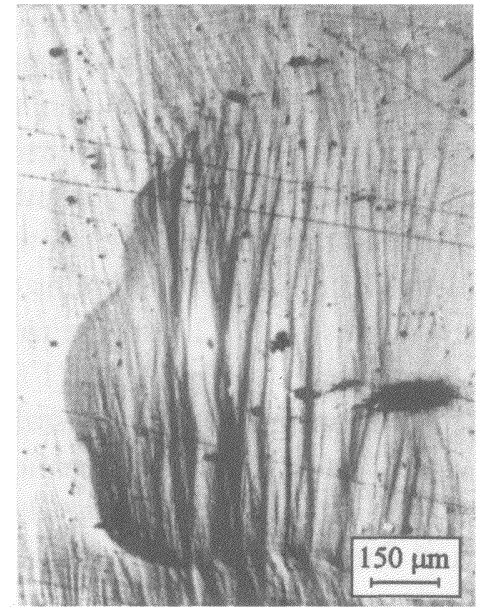

a

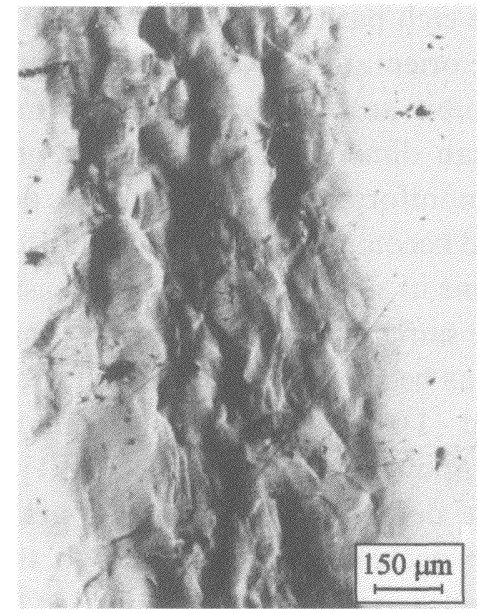

b

FIGURE 8 Bands in a zone of fatigue extrusion: (a) coarse-grains polycrystal; (b) fine-grains polycrystal. 


\section{The Effect of the Banded Structures in the Deformed Material on the Structure and the Texture of the Recrystallized State}

The banded features - shear bands, transition bands and twins - are the sites where recrystallization centers are formed preferably (Fig. 9). In the coarse-grain polycrystals most of recrystallization centers also appear in the said bands. However, when the grain dimensions are decreased, the centers are formed at the grain boundaries, too. This is probably due to the boundary slip of the grains and their rotation. The preferable formation of recrystallization centers in shear and transition bands determines the structure and the texture of the recrystallized material. These bands have a certain set of orientations. For example, the transition bands, which are produced in rolled crystals having the initial orientation (1 110$)\left[\begin{array}{lll}0 & 0 & 1\end{array}\right]$, contain microbands with the orientations from $\left\{\begin{array}{lll}1 & 0\end{array}\right\}$ to $\left\{\begin{array}{lll}1 & 1\end{array}\right\}$. As follows from the findings described above (see also Fig. 5), the shear bands in a deformed single crystal having the stable orientation (1 111$)\left[\begin{array}{lll}0 & \overline{1} & 1\end{array}\right]$ may contain microbands with the orientations from $\left\{\begin{array}{llll}1 & 1 & 1\end{array}\right\}$ to $\left\{\begin{array}{lll}5 & 1 & 1\end{array}\right\}$.

It is known that particular annealing conditions may determine the "choice" of the recrystallization centers being realized. A slow-heating annealing of a deformed single crystal $\left(\begin{array}{lll}1 & 1 & 0\end{array}\right)\left[\begin{array}{lll}0 & 0 & 1\end{array}\right]$ produces a recrystallization texture $\left\{\begin{array}{lll}3 & 1 & 0\end{array}\right\}\langle 001\rangle-\{210\}\langle 001\rangle$, while a rapid-heating annealing results in a sharp texture $\left(\begin{array}{llll}1 & 1 & 0\end{array}\right)\left[\begin{array}{lll}0 & 0 & 1\end{array}\right]$.

Recently Gubernatorov et al. (1995) and Sokolov et al. (1995) have proposed that the orientation of possible recrystallization centers is

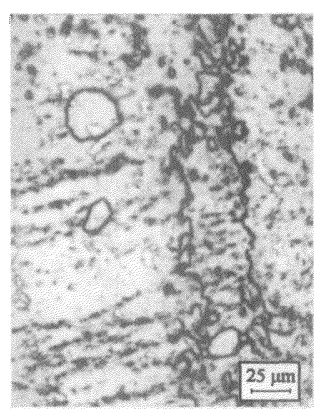

a

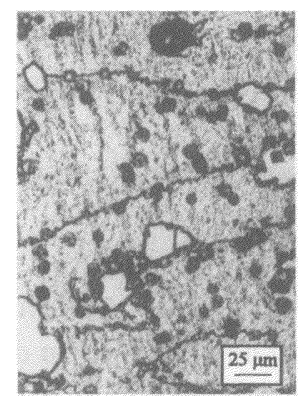

b

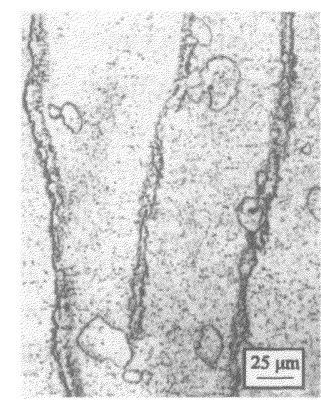

C

FIGURE 9 Forming of the recrystallization grains on the bands of rolled single crystals: (a) on the shear bands; (b) on the transition bands; (c) on the twins. 
limited by the critical dimension of the nuclei, which depends on the orientation relation between potentially possible nuclei and the matrix.

When the deformed material is heated slowly, the centers enclosed by the boundary with a minimum surface energy are realized first. These centers grow at a slower rate than the potential centers enclosed by highenergy boundaries. However, the former centers may grow to have large dimensions under slow-heating annealing conditions. In this situation the centers having high-energy boundaries are not realized, because their critical dimension should be very large.

Thus, the bands in the deformed material provide a certain (limited) set of orientations of possible recrystallization centers. Orientations of the really formed centers (and, correspondingly, the recrystallization texture) depend, in addition, on particular annealing conditions.

The number of different types of the bands may determine to a certain extent the number of recrystallization centers. In these terms one may speak about the effect of the structure of the deformed state on the structure of the recrystallized material.

\section{The Effect of the Rolling Factors on the Structure of the Deformed Material}

In terms of the continuum mechanics rolling is treated as a simple deformation under the action of a system of compression (normal to the rolling plane) and tension (in the rolling direction) stresses. In actual fact, however, the rolled material has a largely nonuniform distribution of stresses, which reflects a complicated pattern of the deformation center and imposes constraints on the form changing. These problems have been discussed in the paper by Gubernatorov et al. (1995).

We shall restrict ourselves to some examples, which illustrate the effect of certain rolling factors on the structure of deformed single crystals.

The study of the structure of the transverse layers of the rolled samples shows that frequently the deformation bands are located in the near-surface regions only. The nonuniform distribution of these bands may be decreased or increased by changing the rolling conditions.

In their recent paper Sokolov et al. (1995) have already discussed this issue. The number of passes and the friction coefficient between the material and the rolls may considerably affect the structure of deformed 
single crystals (Fig. 10(a) and (b)). When the number of passes and the friction coefficient are increased during rolling of the single crys-

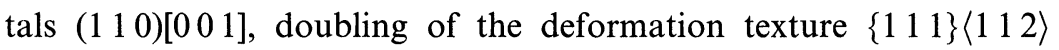
decreases. It is possible to produce a strictly one-component texture. In this case the structure of the deformed single crystal is free of deformation and transition bands and contains shear bands only.

Figure 10 shows the structure of a single crystal (1 110$)\left[\begin{array}{lll}0 & 0 & 1\end{array}\right]$, which was deformed in rolls having small (c) and large (d) diameters. It is seen that a high-rolling pressure (large-diameter rolls) causes refinement of the banded structure in the deformed material.

As was mentioned in the foregoing, the banded structure formed in the boundary regions of deformed coarse-grain polycrystals differs to a great extent from the structure in the central regions of these crystals. This suggests that the evolution of the bands under deformation is determined by the slip scheme at the very beginning of the form changing by the material.
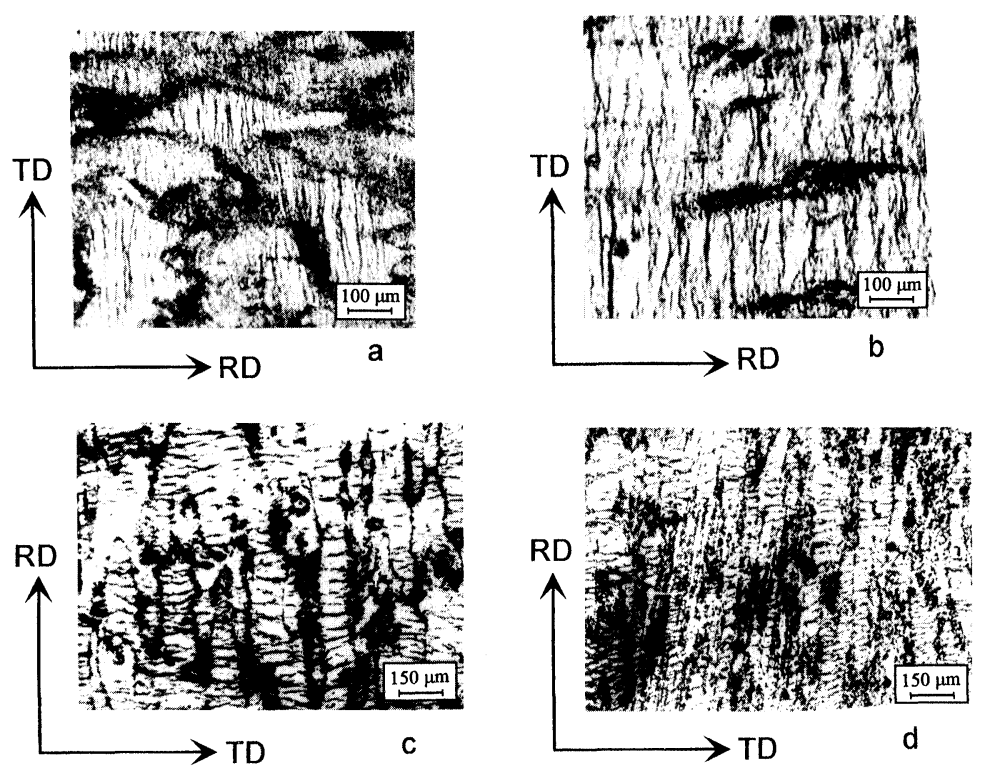

FIGURE 10 The effect rolling factors on the bands structure of deformed single

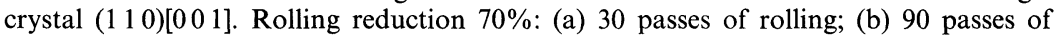
rolling with high interfacial friction; (c) the deformation in rolls having diameter $55 \mathrm{~mm}, 8$ passes of rolling; (d) the deformation in rolls having diameter $180 \mathrm{~mm}, 8$ passes of rolling. 


\section{RESEARCH RESULTS AND DISCUSSION}

This study has yielded the following results:

1. A nonuniform distribution of coarse traces of the slip is revealed even at early stages of the deformation (5-10\%) of the single crystals. The single crystals are broken up into regions $30-150 \mu \mathrm{m}$ in size. The slip in every region differs slightly from the slip in the adjacent region. The orientation of the single crystals is scattered by $2-8^{\circ}$.

2. If the deformation is increased $15-20 \%$, these regions extend in the rolling direction. Single crystals having unstable orientations contain, along with the initial orientation, the texture components, which differ from the initial orientation.

3. At a deformation of $20-25 \%$, deformation bands bounded by regions of the structure having the initial orientation appear. If the degree of deformation is increased further, these regions become narrow and turn into transition bands. Indicators of the deformation bands with different orientations are also observed in single crystals having stable orientations. However, the boundary between these bands is not always revealed by etching.

4. Deformation bands with narrow transition bands are well-detected after rolling with a $30 \%$ or heavier reduction. The number of the bands does not increase at higher degrees of deformation.

5. The deformation of about $20 \%$ gives rise to shear bands. They are detected first in the regions having the initial orientation. The number of the shear bands grows with increasing deformation.

6. In the single crystals studied traces of the shear bands in the rolling plane are generally parallel to the transverse direction and those on the side surfaces make an angle of $25-45^{\circ}$ with the rolling direction. The shear planes have versatile crystallographic indices and do not coincide with the active slip planes.

7. The banded structures are formed in coarse-grains of the rolled polycrystals similarly to those in single crystals having the corresponding orientations. The exception is the boundary regions where the banded structure is distorted markedly. If the dimensions of the initial grains decrease, the regions of the distorted banded structure cover the whole grain. In addition, shear bands, which cover groups of several grains, are observed. 
8. When the alloy was subject to fatigue tests, the structural pattern of the development of the banded structures in the extrusion areas fully corresponded to the pattern, which was observed after rolling.

9. Recrystallization centers are formed during annealing preferably in shear bands, transition bands and twins. The annealing heating rate affects the orientation of the recrystallization centers, which appear preferably in the banded structures.

10. The rolling factors have a considerable effect on the number and structure of the bands and also on the texture formed in the material during deformation.

These findings confirm that the appearance of deformation and shear bands is directly connected with the deformation nonuniformity. This nonuniformity may be due, on the one hand, to a nonuniformity of the initial state of the material and, on the other hand, to a nonuniform distribution of the stresses, which are determined by the scheme of the deformation zone and constraints on the material flow.

Undoubtedly, the shear bands appear as a result of strengthening the deformed material and the impossibility for the deformation to proceed further by the ordinary slip. Strengthening is higher in structurally inhomogeneous materials and single crystals containing impurities, inclusions or twins.

The shear bands lead to disordering so that deformation may proceed further under the same loads. Thus, the formation of the bands is considered as the loss of stability by the hardened material.

Seemingly, if strengthening is low and the stresses may relax during deformation, the form of the material could change without the formation of the banded structures. For example, in the single crystal $\left\{\begin{array}{lll}1 & 0 & 0\end{array}\right\}\left\langle\begin{array}{lll}0 & 1 & 1\end{array}\right\rangle$ their formation is suppressed but is not prohibited. The formation of the bands is independent of the crystallographic stability of the crystal orientations with respect to deformation. This inference is supported, for example, by the structure of the deformed single crystal $\left\{\begin{array}{llllll}1 & 1 & 1\end{array}\right\}\left\langle\begin{array}{llll}0 & 1 & 1\rangle & 1\end{array}\right.$.

This study has yielded one more significant result: the orientation of the deformed material changes in longitudinal and transverse layers. However, it is not always possible to reveal the boundary between these layers by etching. Even if the orientation is stable, the deformed single crystals exhibit longitudinal bands having a slightly different 
orientation. However, transition bands cannot be detected by metallographic etching.

Discussing the nature of the shear bands, it should be emphasized that a significant indication of these bands is their regular arrangement with respect to the acting forces (in the case of rolling, the surfaces bear traces perpendicular to the deformation direction in the rolling plane and inclined $30-40^{\circ}$ in the lateral cross-section). These surfaces have absolutely different crystallographies.

It is worth noting that the modern shear models cannot explain all the experimental results. These models are based on the following concepts:

- Consideration of the geometry of the maximum shear stresses (Schmid and Taylor factors).

- Consideration of the principle of the minimum plastic work of deformation by means of the Taylor factor allowing for the deformation efficiency.

- Consideration of the work hardening rate and the geometrical softening factor achieved after rotation of the lattice of the deformed material.

- The slip plane minimum principle.

The analysis of the shear models (for example, the study by Lee and Chan (1991)) shows that calculated planes do not always coincide with the observed planes. Refined shear models allow for an ever increasing number of constraints. This makes them cumbersome and inconvenient for calculations.

The concepts of the structure instability in strongly excited states have been largely accepted quite recently. They have been proposed by Likhachov et al. (1989) and have been currently developed by Academician Panin's school of thought (1995). In accordance with these principles, the nucleation of the plastic shear is a local structural transition described in terms of the nonequilibrium thermodynamics. The mechanical field within a crystal propagates in displacement and rotation waves (rotation-shear vortices), while the material continuum is preserved. The regions of localization of large stresses (and strains) turn into regions of self-excited states. They can emit defects, which cause self-excitation of the neighboring regions (a relay excitation).

The self-excitation is the source of the meso- and macroplastic instability. It may lead to a quasiviscous flow of the material (as with 
hydraulic compression). The ensemble of the defects emitted from the excited regions distorts the crystal lattice and alters the usual regular features of slip and shear. These concepts are very similar to the hypothesis of softening due to a local adiabatic heating, but they stem from deformation. The researchers believe this theory is verified by a certain layout of the bands at different crystallographies of the material, analogous regularities by which the bands are formed in noncrystalline materials (plastics, organic compounds), amorphization of the shear areas during plastic deformation of some materials, and impartment of the local deformation to neighboring crystallites in polycrystals.

Considering the above discussion, the study of the formation of the bands of these types should be focused on the stress distribution over the material, the stress nonuniformity, and the deformation constraints.

\section{References}

Gubernatorov, V.V., Sokolov, B.K. and Sbitnev, A.K. (1995) On the texture formation in metals during recrystallization. I. The effect of the texture on the relative grain growth rate. Fiz. Met. Metalloved. 81(3), 44-52.

Lee, W.B. and Chan, K.C. (1991) A criterion for the prediction of shear band angles in F.C.C. metals. Acta Metall. Mater. 39(3), 411-417.

Likhachov, V.A., Panin, V.E., Zasimchuk, E.Z., Vladimirov, V.I., Romanov, A.E., Gorskii, V.V. et al. (1989) Cooperative Deformation Processes and Deformation Localization. Kiev, Naukova Dumka, p. 320.

Panin, V.E., Yegorushkin, V.Ye., Makarov, P.V., Grinajev, Y.V., Popov, V.L., Derugin, E.E. et al. (1995) Physical Mesomechanics and Computer-Aided Design of Materials. In two volumes. Editor Acad. V.E. Panin. Novosibirsk, Nauka, Siberian Publishing Firm RAS, pp. 298; 240.

Sokolov, B.K., Sbitnev, A.K., Gubernatorov, V.V., Gervasyeva, I.V. and Vladimirov, L.R. (1995) On the influence of the annealing heating rate on the recrystallization texture of a deformed single crystal $\left(\begin{array}{lll}1 & 1 & 0\end{array}\right)\left[\begin{array}{lll}0 & 0 & 1\end{array}\right]$ of $3 \%$ silicon iron. Textures and Microstructures 26-27, 427-443. 